\title{
A MULTI-LAYERED POTENTIAL FIELD METHOD FOR WATER- JET PROPELLED UNMANNED SURFACE VEHICLE LOCAL PATH PLANNING WITH MINIMUM ENERGY CONSUMPTION
}

\author{
Shasha Wang \\ Mingyu Fu \\ Yuanhui Wang \\ Liangbo Zhao \\ College of Automation, Harbin Engineering University, China
}

\begin{abstract}
Focusing on the influence of wind and surface currents on local path planning in the marine environment, a multilayered potential field (MPF) method is proposed to minimize the energy consumption of a water-jet propelled unmanned surface vehicle (USV). A synthetic environment framework that can incorporate the information of the base potential field layer and the environment layer is constructed first. This framework provides a base for minimizing the energy consumption of the water-jet propelled USV through proper force weighting. For the purpose of USV path planning, the traditional potential field method is extended by including the velocity information of the USV and the approached obstacles to avoid collisions with dynamic obstacles. The proposed method integrates kinematic control to prevent considering the vehicle as a point mass or rigid body. Finally, simulation and comparison experiments are performed to demonstrate the energy-saving efficiency of the proposed local path planning approach for the water-jet propelled USV.
\end{abstract}

Keywords: water-jet propelled USV, local path planning, marine environment, minimum energy consumption, multi-layered potential field method

\section{INTRODUCTION}

The USVs have been widely used in various marine applications in the past decades, with military applications being dominant. When the USVs perform dangerous missions, such as sea patrol or coastal guarding in harsh environments, the risk to the personnel is obviously reduced due to the minimal involvement of human operators [1]. In addition, it should be noted that civilian applications, such as environmental monitoring [2] or search and rescue missions [3], are also important and promising for the USVs. In highly polluted lakes, USVs can be used to collect water samples without direct human participation [2]. Together with a micro aerial vehicle, the USV played an important role while surveying damages in parts of Marco Island after Hurricane Wilma in 2005 [3]. Therefore, USVs can be combined with other unmanned systems to perform various tasks without human intervention, and essentially exhibit highly nonlinear dynamics.

Path planning aims to guide the vehicle from its initial position to the destination while possessing accurate perception of the environment and effective collision avoidance capabilities [4]-[6]. The path planning process can generally have the form of three different approaches: global approach (path generated prior to launching the USV) [7], local approach (path generated while the vehicle is en-route) [8], and hybrid approach [9]. To obtain the path, different computational methods can be applied, such as optimization 
methods based on evolutionary algorithms (EAs) and/or genetic algorithms (Gas) [10]), heuristic search algorithms (graph search techniques), and potential fields (artificial potential fields - APFs). However, GAs has the drawbacks of lacking convergence and expensive computational costs. Thus the generated path may lack consistency, which will limit its real-time implementation. Compared to optimization methods, the heuristic methods (graph search techniques) can quickly find an optimal path with the least number of nodes because they use the grid-based strategy [11][12]. However, a further path smoothing procedure is needed to deal with the non-holonomic constraints of the vehicle [11]. Moreover, they don't provide the global optimal path and are not suited to cases where the environment is highly dynamic and has strong effects on the robots.

In the potential field (PF) approach, the target points are assigned with attractive fields, while the obstacles are distributed with repulsive fields [13]. This approach is becoming more and more popular, due to its easy implementation and good collision avoidance capability. The input data to the potential field algorithm can be obtained directly from range sensors, without map calculations and estimations. Potential field algorithms are appropriate for underlying real-time control at the lack of global information, but the problem of local minima and target unreachability may appear. Therefore, this kind of problem needs to be handled by global algorithms at a higher layer [13]. Here, two major solutions have been worked out [14][15]. When facing moving obstacles and the time-varying environment, the APF can modify the path to avoid collisions. However, most potential field methods focus their attention on theoretical analyses and regard the vehicle as a mass point or rigid body without constraints. Moreover, the marine environment and the limited energy of the vehicle are not sufficiently taken into account or even ignored in path planning [16].

The influence of the marine environment cannot be ignored when developing the path planning algorithms for marine vehicles, especially for those with limited operating speed and relatively small dimensions and displacements. The environmental effects and energy consumption should be considered in the path planning in the ocean, which is different from the general path planning on land, to find the shortest path while avoiding the obstacles [17]. Although many researchers have proposed intelligent path planning algorithms to deal with the environmental influences of autonomous underwater vehicles (AUVs), very little work has been done for unmanned surface vehicles (USVs). Thus, if the generated path ignores the environmental effects, more fuel may be consumed, or the path may be too difficult to follow [18]. It is difficult to select the optimal path in terms of the expected energy consumption using only the kinematics-based or distance-based approach. In [19], Garau et al. presented the effect of current fields in the path planning problem using the $\mathrm{A}^{\star}$ search algorithm with time-optimal cost. They emphasized the importance of the marine environment in path planning. Considering the time-varying currents, an iterative non-linear optimization method was used in [20] to find the time-optimal path. The comparison has also been made with other selected path planning algorithms, such as RRT, Line-of-sight, $A^{*}$, and Constant-Time Surfacing- $A^{*}$ [21]. In addition, numerous energy-efficient path planning approaches have been applied to improve the endurance of surface vehicles. However, non-holonomic constraints of vehicles were ignored in those approaches [22]-[24].

In view of the above issues, this work is devoted to developing a multi-layered potential field method for a waterjet propelled USV to minimize its energy consumption in the presence of marine environments, such as wind and surface currents. The diagram of the method is shown in Fig. 2. The performance of the proposed algorithm was verified through comparison of simulation results. The main contributions of the paper can be summarized as below:

1) Taking into account the influence of the wind and surface currents on the local path planning of a water-jet propelled USV, a multi-layered potential field method is proposed to minimize the energy consumption.

2) The paper constructs a synthetic environment framework, which incorporates the information of the base potential field layer and the environment layer.

3) The traditional potential field method is extended by including the velocity information of the USV and the obstacles to avoid collisions with dynamic obstacles.

\section{PLANNING SPACE REPRESENTATION}

\section{PROBLEM STATEMENT}

To achieve autonomous USV path planning in the maritime environment, two critical problems need to be addressed. The first problem is collision avoidance with dynamic obstacles composing kinematic constraints. The second problem is generation of the minimum energy consumption path under the influence of wind and surface currents. To address the aforementioned problems and challenges, a synthetic environment framework and multi-layered potential field method are developed in this paper.

Assumption 1: The position and velocity of both the USV and the target are well known.

Assumption 2: The positions and velocities of surface obstacles can be accurately measured online.

Assumption 3: Among the marine environmental effects, wind and surface currents are mainly considered in this paper.

Assumption 4: The obstacle velocity is equal to or less than the USV velocity, and its motion is linear and uniform.

\section{KINEMATIC MODEL OF WATER-JET PROPELLED USV}

The USV is equipped with water-jet propulsion, nozzle pump, rudder sensor, astern pump \& sensor, compass, radar, GPS, anemoscope \& dogvane, wireless system, etc. [25]. The equipment of the USV is shown in Fig. 1. To simplify the algorithm, the USV is assumed to be a three degree of 
freedom (3-DOF) system, including surge, sway, and yaw [1]. Then, the kinematic model is formulated as:

$$
\dot{\eta}=J(\eta) v=\left\{\begin{array}{l}
\dot{x}=u \cos (\psi)-v \sin (\psi) \\
\dot{y}=u \sin (\psi)+v \cos (\psi) \\
\dot{\psi}=r
\end{array}\right.
$$

where $\eta=[x, y, \psi]^{\mathrm{T}}$ is the position and heading vector with coordinates in the earth-fixed reference frame ( $\{\mathrm{E}\}$-frame), and $v=[u, v, r]^{T}$ is the linear and angular velocity vector with coordinates in the body-fixed reference frame ( $\{\mathrm{B}\}$-frame).

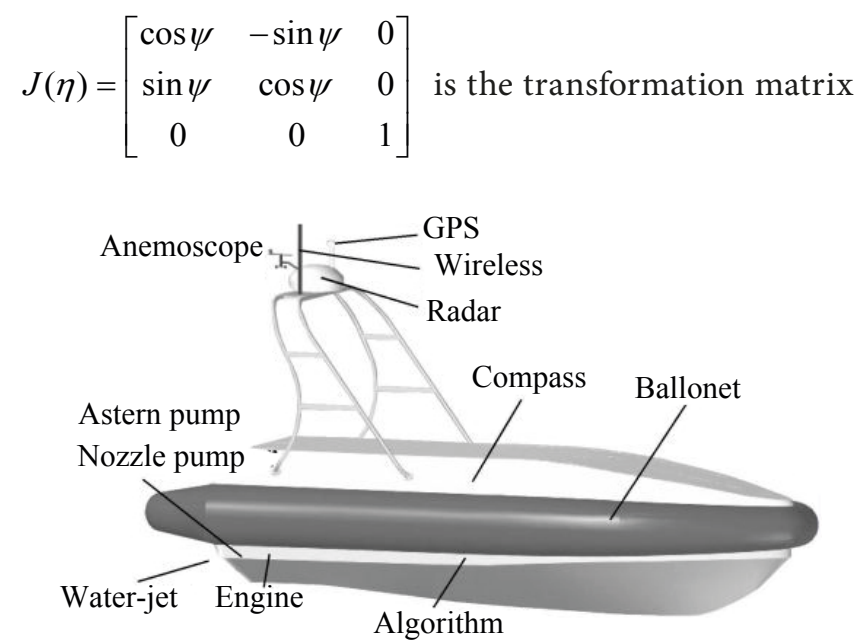

Fig.1. The equipment of water-jet propelled USV

The above condition can be equivalently formulated as

$$
\left\{\begin{array}{l}
\dot{x}=u_{\text {total }} \cos (\psi+\beta) \\
\dot{y}=u_{\text {total }} \sin (\psi+\beta) \\
\dot{\psi}=r
\end{array}\right.
$$

where, $u_{\text {total }}=u_{t o l}=\sqrt{u^{2}+v^{2}}$ is the resultant velocity for USV, and $\beta=\arctan (v / u)$ is the sideslip angle.

\section{ENERGY CONSUMPTION MODEL OF WATER-JET PROPELLED USV}

The propulsion adopts the water jet HJ292, made by Hamilton Company, the detailed parameters of which are listed in Appendix, Tab. 3. The output power $P_{e}$ of the waterjet propeller is [26]:

$$
P_{e}=\rho g A_{j} v_{j}\left[\frac{v_{j}^{2}}{2 g}+h_{s}+h_{j}-\zeta\left(\frac{v_{s}^{2}}{2 g}\right)\right] / \eta_{s} \eta_{p}
$$

where $g$ is the acceleration of gravity; $\rho$ is the sea water density; $A_{j}, A_{i n}, A_{w}$ are the cross sectional areas of nozzle, water inlet, and bend, respectively; $v_{j}$ is the nozzle velocity; $v_{s}$ is the inlet velocity; $h_{s}$ is the water-jet propeller loss; $h_{j}$ is the vertical distance between inlet and water-jet center, and $\zeta$ is the kinetic energy utilization coefficient.

The nozzle velocity $v_{j}$ is:

$v_{j}=-\left[\left[-\frac{q}{2}-\left(\left(\frac{q}{2}\right)^{2}+\left(\frac{p}{3}\right)^{3}\right)^{\frac{1}{2}}\right]^{\frac{1}{3}}+\left[-\frac{q}{2}+\left(\left(\frac{q}{2}\right)^{2}+\left(\frac{p}{3}\right)^{3}\right)^{\frac{1}{2}}\right]^{\frac{1}{3}}\right]$

where $v_{s}=u_{\text {total }}$ and

$$
\left\{\begin{array}{c}
p=g \frac{h_{j}-\zeta \frac{v_{s}^{2}}{2 g}}{\frac{1}{2}+\frac{e_{i n}}{2}\left(\frac{A_{j}}{A_{i n}}\right)^{2}+\frac{e_{w}}{2}\left(\frac{A_{j}}{A_{w}}\right)^{2}+\frac{e_{j}}{2}} \\
q=\frac{-C\left(\frac{n}{1000}\right)^{3} \eta_{p}}{\rho A_{j}\left[\frac{1}{2}+\frac{e_{i n}}{2}\left(\frac{A_{j}}{A_{i n}}\right)^{2}+\frac{e_{w}}{2}\left(\frac{A_{j}}{A_{w}}\right)^{2}+\frac{e_{j}}{2}\right]}
\end{array}\right.
$$

The actual energy consumption $W_{t}$ of the water-jet propelled USV is expressed as:

$$
W_{t}=\sum_{i=1}^{N} d_{i} P_{e} / u_{t o l}
$$

where $d_{i}$ is the length of $i_{\text {th }}$ path segment during $\Delta t$, and $N$ is the total number of path segments.

\section{ENVIRONMENT FRAMEWORK}

In the majority of previous works, the path planning algorithm has been primarily applied in the configuration space ( $C$-space), which consists of the obstacle space $\left(C_{o b s}\right)$ and the collision-free space ( $\left.C_{\text {free }}\right)$. Within the $C$-space, the movement of the USV can be represented as the configuration, i.e. the vector specifying all USV motion parameters, such as its position and orientation [26]. In general, the $C$ space is the set of all possible configurations.

A new environment framework is constructed in the form of a synthetic field representing the influence of both the dynamic obstacles and the marine environment. This framework bears the name of the synthetic force function $F_{\text {syn }}$ and includes two main layers (see Fig. 2):

1) The $C$-space is the base potential field layer, denoted as $U_{\text {base }}$, which can be obtained from the data acquisition module of the USV navigation system [16]. Normally, the base potential field layer is represented as a binary grid map, where the obstacles and the collision-free space are distinguished in black and white. In each grid, the configuration has its coordinates relative to the $C$-space frame coordinates, and the corresponding weights with values of $0\left(C_{o b s}\right)$ or $1\left(C_{\text {free }}\right)$. 
2) The marine environment influence factors are represented as the environment layer, denoted as the environment force function $F_{e n v}$. The ocean wave effect is not negligible in terms of force magnitude, but the frequency range does not affect vehicle maneuvering, and the marine waves mostly affect the floating body motions, which are more important for dynamic positioning than for path planning. According to Assumptions 2 and 3, this paper mainly considers the influence of wind and surface currents which are known in advance.

\section{MULTI-LAYERED POTENTIAL FIELD METHOD}

A multi-layered potential field method is proposed for planning the path of the water-jet propelled USV to minimize its energy consumption, considering the environmental impact of the wind and surface currents. Unlike conventional potential functions, the here proposed multi-layered potential field method constructs a synthetic environment framework which incorporates the information of the base potential field layer and the environment layer to generate the energy-saving efficiency path. In addition, the proposed method integrates kinematic control, and the attractive and repulsive functions are extended by including the velocity information of the USV and the obstacles to avoid collisions with dynamic obstacles.

\section{ALGORITHM STRUCTURE}

Fig. 2 outlines the schematic of the multi-layered potential field approach. The pre-processed synthetic field map is generated from the two-layered information. The first layer is called the base potential field layer and represents the general navigation information, which mainly reflects the influence of obstacles. The base potential field layer has two sub-layers: 1) the attraction potential field, on which the target point of the path is given as the specific mission requirement, and 2) the repulsion potential field, where the information of dynamic obstacles, for example moving vehicles, is calculated from the sensor data.

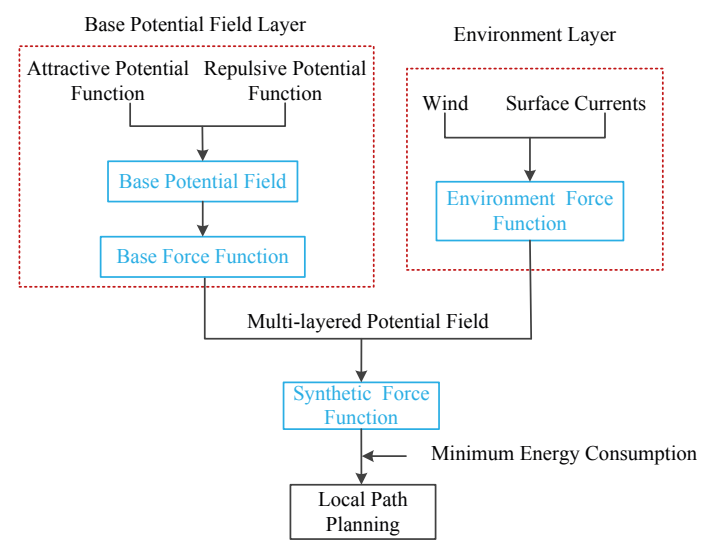

Fig.2. Schematic of the multi-layered potential field method
The second layer bears the name of the environment layer. It contains sub-layers with all the information of marine environmental factors, such as wind and surface currents, that can affect the USV voyage. All the information in the base potential field and the environment layers is used to search for a path. Details on how to obtain the synthetic field and the synthetic force function, as well as the minimum energy consumption strategies will be illustrated in the following sections.

\section{BASE POTENTIAL FIELD LAYER}

\section{1) Attractive potential function}

The conventional potential field method defines the attractive potential function as a function of the relative distance between the USV and the target, regardless of the USV velocity. Hence, the conventional method sometimes fails to provide a soft-landing solution, more precisely: the USV reaches the target with non-zero speed. To overcome this difficulty, the attractive potential function given by Eq. (7) is proposed [16]. This function contains the velocity information which provides soft landing. The idea of taking the velocity into account has been adapted in the following way:

$$
U_{\text {att }}\left(d_{P G}, u_{t o l}\right)=\lambda_{1} d_{P G}(t)^{m}+\lambda_{2}\left\|u_{t o l}(t)\right\|^{n}
$$

where $d_{P G}(t)$ is the Euclidean distance between the USV and the target, $u_{\text {tol }}(t)$ is the USV velocity at time $t ; \lambda_{1}, \lambda_{2}$ are positive scalar parameters; and $m, n$ are positive constants.

The main disadvantage of the above attractive potential function is that the attraction force significantly depends on the distance to the target. In order to solve this problem, the method makes use of some improvements. The benefit of the improved function is that the attractive potential field is almost constant, as a result of which the generated path is independent from the distance to the target when the USV is not close to it and the function can avoid the local minimum problem.

$$
U_{a t t}\left(d_{P G}, u_{t o l}\right)=F(P)\left(\frac{\alpha}{\beta^{2}} d_{P G}+\frac{\alpha}{\beta+d_{P G}}-\frac{\alpha}{\beta}+\lambda_{2}\left\|u_{t o l}\right\|^{2}\right)
$$

where $\alpha, \beta$ are positive constants. The term $\lambda_{2}\left\|u_{t o l}(t)\right\|^{2}$ regarding the velocity is chosen to be quadratic. $F(P)$ is the rotation matrix defined as:

$$
F(P)=\left[\begin{array}{cc}
\cos \theta & \sin \theta \\
-\sin \theta & \cos \theta
\end{array}\right]\left[\begin{array}{cc}
\cos \left(B(P)^{*} \theta\right) & -\sin \left(B(P)^{*} \theta\right) \\
\sin \left(B(P)^{*} \theta\right) & \cos \left(B(P)^{*} \theta\right)
\end{array}\right]\left[\begin{array}{cc}
\cos \theta & -\sin \theta \\
\sin \theta & \cos \theta
\end{array}\right]
$$

The rotation matrix describes the nature of USV operations to be performed in order to avoid collisions and local minima efficiently.

In Eq. (9), $B(P)$ is $B(P)=e^{\left(-H(P)^{4}\right)} ; H(P)$ is the resultant force; $B(P)$ is the decay function, and $q=2$ is the decay 
rate. When the magnitude of the resultant force approaches zero, the value of $B(P)$ is close to one. And as $H(P)$ grows, $B(P)$ decays rapidly to zero.

It can be seen that $\frac{\alpha}{\beta^{2}} d_{P G}+\frac{\alpha}{\beta+d_{P G}}-\frac{\alpha}{\beta}$ and $\lambda_{2}\left\|u_{t o l}\right\|^{2}$ are both non-negative. This means that $U_{a t t}\left(d_{P G}, u_{t o l}\right)$ approaches its minimum of zero if and only if both the USV velocity and the distance between the target and the USV approach zero.

The attractive force vector function pointing from the USV to the target can be calculated as the derivative of the above potential function:

$$
\begin{aligned}
& \mathbf{F}_{a t t}\left(d_{P G}, u_{t o l}\right)=-\nabla U_{a t t}\left(d_{P G}, u_{t o l}\right) \\
& =F(P)\left(\frac{\partial U_{a t t}\left(d_{P G}, u_{t o l}\right)}{\partial d_{P G}} \mathbf{n}_{P G}+\frac{\partial U_{a t t}\left(d_{P G}, u_{t o l}\right)}{\partial u_{t o l}} \mathbf{n}_{V P G}\right)
\end{aligned}
$$

where $\mathbf{n}_{P G}$ is the unit vector pointing from the USV to the target and $\mathbf{n}_{V P G}$ is the unit vector pointing in the USV velocity direction.

$$
\begin{gathered}
\frac{\partial U_{a t t}\left(d_{P G}, u_{t o l}\right)}{\partial d_{P G}}=\frac{\alpha}{\beta^{2}}-\frac{\alpha}{(\beta+x)^{2}} \\
\frac{\partial U_{a t t}\left(d_{P G}, u_{t o l}\right)}{\partial u_{t o l}}=2 \lambda_{2}\left\|u_{t o l}\right\| \\
\mathbf{F}_{a t t}\left(d_{P G}, u_{t o l}\right)=\left(\left(\frac{\alpha}{\beta^{2}}-\frac{\alpha}{\left(\beta+d_{P G}\right)^{2}}\right) \mathbf{n}_{P G}+\right. \\
\left.+2 \lambda_{2}\left\|u_{t o l}\right\| \mathbf{n}_{V P G}\right) F(P)
\end{gathered}
$$

The attractive force functions are shown in Fig. 3. When the velocity is constant, the force field proposed in this paper is almost constant when the USV is relatively far from the target. The force vector function in Fig. 3 is given by:

$$
\begin{gathered}
\mathbf{F}_{a t M A X}\left(u_{t o l}\right)=\left.F_{a t t}\left(d_{P G}, u_{t o l}\right)\right|_{d_{P G} \rightarrow \infty}=\frac{\alpha}{\beta^{2}} \mathbf{n}_{P G}+2 \lambda_{2}\left\|u_{t o l}\right\| \mathbf{n}_{V P G} \\
\mathbf{F}_{a t t M I N}\left(u_{t o l}\right)=\left.F_{a t t}\left(d_{P G}, u_{t o l}\right)\right|_{d_{P G} \rightarrow 0}=2 \lambda_{2}\left\|u_{t o l}\right\| \mathbf{n}_{V P G}
\end{gathered}
$$

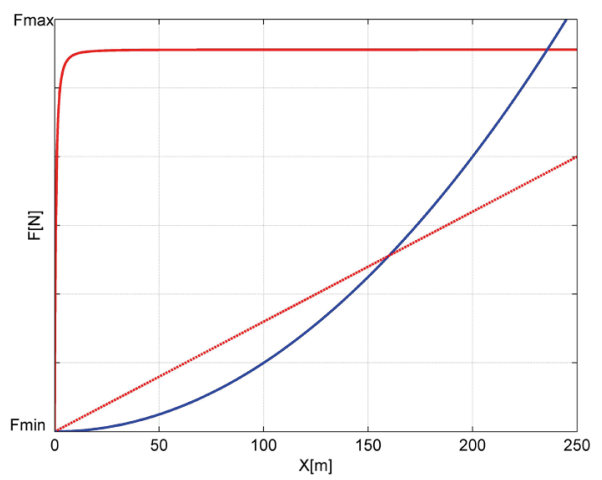

Fig.3. The improved attractive force function according to Eqs., (red solid line), and the linear and exponential characteristics of attractive force functions widely used in the literature, e.g. Eq. (7) (blue solid line and red dashed line, respectively).

\section{2) Repulsive potential function}

In order to avoid collisions with dynamic obstacles while taking the velocities of the USV and the obstacles into account, the repulsive potential function is proposed in the following form:

$$
\begin{aligned}
& U_{\text {rep }}\left(d_{P O}, u_{t o l}, u_{o b s}\right) \\
& =\left\{\begin{array}{cr}
\frac{1}{2} \lambda_{3}\left(\frac{1}{d_{P O}}-\frac{1}{d_{0}}\right)^{2} d_{P G}^{2}+\frac{1}{2} \lambda_{4}\left\|u_{t o l}-u_{o b s}\right\|^{2}, & d_{P O}<d_{0} \\
\frac{1}{2} \lambda_{4}\left\|u_{t o l}-u_{o b s}\right\|^{2} & , d_{P O} \geq d_{0}
\end{array}\right.
\end{aligned}
$$

where $d_{P O}$ is the Euclidean distance between the USV and the obstacle, $u_{\text {obs }}$ is the velocity of the dynamic obstacle; $\lambda_{3}, \lambda_{4}$ are scalar positive parameters; $d_{0}$ is the positive constant denoting the distance of influence of the obstacle.

The repulsive force vector function pointing from the USV to the obstacle can be calculated as the derivative of the potential function:

$$
\begin{aligned}
& \mathbf{F}_{r e p}\left(d_{P O}, u_{t o l}, u_{o b s}\right)=-\nabla U_{r e p}\left(d_{P O}, u_{t o l}, u_{o b s}\right) \\
& =\frac{\partial U_{r e p}\left(d_{P O}, u_{t o l}, u_{o b s}\right)}{\partial d_{P O}} \mathbf{n}_{P O}+\frac{\partial U_{r e p}\left(d_{P O}, u_{t o l}, u_{o b s}\right)}{\partial\left(u_{t o l}-u_{o b s}\right)} \mathbf{n}_{V P O}
\end{aligned}
$$

where $\mathbf{n}_{P O}$ is the unit vector pointing from the USV to the obstacle and $\mathbf{n}_{V P O}$ is the unit vector pointing in the direction of the relative USV velocity with respect to the dynamic obstacle.

$$
\begin{gathered}
\frac{\partial U_{r e p}\left(d_{P O}, u_{t o l}, u_{o b s}\right)}{\partial d_{P O}}=\lambda_{3}\left(\frac{1}{d_{P O}}-\frac{1}{d_{0}}\right) d_{P O}^{-2} d_{P G}^{2} \\
\frac{\partial U_{r e p}\left(d_{P O}, u_{t o l}, u_{o b s}\right)}{\partial\left(u_{t o l}-u_{o b s}\right)}=\lambda_{4}\left\|u_{t o l}-u_{o b s}\right\|
\end{gathered}
$$

$\mathbf{F}_{\text {rep }}\left(d_{P O}, u_{t o l}, u_{o b s}\right)$

$= \begin{cases}\lambda_{3}\left(\frac{1}{d_{P O}}-\frac{1}{d_{0}}\right) d_{P O}^{-2} d_{P G}^{2} \mathbf{n}_{P O}+\lambda_{4}\left\|u_{t o l}-u_{o b s}\right\| \mathbf{n}_{V P O}, d_{P O}<d_{0} \\ \lambda_{4}\left\|u_{t o l}-u_{o b s}\right\| \mathbf{n}_{V P O} & , d_{P O} \geq d_{0}\end{cases}$

The base force function $F_{\text {base }}$ is obtained by adding the attractive force function and the repulsive force functions given by Eqs. (12) and (19), respectively.

$$
F_{b a s e}=F_{a t t}\left(d_{P G}, u_{t o l}\right)+\sum_{i=1}^{h} F_{r e p}\left(d_{P O}, u_{t o l}, u_{o b s}\right)
$$

where $i$ is the number of obstacles and varies from 1 to $h$. 


\section{ENVIRONMENT LAYER AND SYNTHETIC FORCE FUNCTION}

The websites of Korea Hydrographic and Oceanographic Administration (KHOA) ${ }^{1}$ in Korea, National Oceanic and Atmospheric Administration (NOAA) ${ }^{2}$ in the United States of America, and the National Centre for Atmospheric Research (NCAR), for instance, provide the marine environmental information. Moreover, data are available from a tidal atlas or admiralty charts for different time periods, based on which different astronomical influences can be predicted. Among them, the prediction of the wind is the most accurate and its actual effect is longer, while the data on surface currents contain uncertainties. Therefore, calculating the surface currents from the wind data is also of practical significance. The surface current velocity $V_{c}$ can be calculated using the Ekman theory as:

$$
V_{c}=\frac{0.0247 V_{w}}{\sqrt{\sin \phi}}
$$

where $V_{w}$ is the wind velocity and $\phi$ is the latitude.

The environment force function is:

$$
F_{\text {env }}=\tau_{\text {wind }}+\tau_{\text {current }}
$$

where $\tau_{\text {wind }}, \tau_{\text {current }}$ are the wind force and the surface currents force, respectively (for detailed calculations, see Appendix A).

Once the base force function and the environment force function are obtained, the synthetic force function $\left(F_{s y n}\right)$ is calculated as a function of $F_{b a s e}$ and $F_{e n v}$ :

$$
F_{s y n}=\varepsilon_{1} F_{e n v}+\varepsilon_{2} F_{b a s e}
$$

where $\varepsilon_{1}$ and $\varepsilon_{2}$ are two force weights for $F_{e n v}$ and $F_{b a s e}$, respectively.

When the USV reaches the target gradually, the $F_{\text {base }}$ decreases continuously and the proportion of the $F_{\text {env }}$ is increasing, which leads to USV concussion at the target point. To address this problem, the $F_{s y n}$ is improved as follows:

$$
F_{s y n}=\varepsilon_{1} F_{e n v} d_{P G}+\varepsilon_{2} F_{\text {base }}
$$

The energy consumption $W_{s y n}$, which is the sum of the synthetic force functions $F_{s y n}$ at each position, can be expressed as:

$$
W_{s y n}=\sum_{i=1}^{N} d_{i} F_{s y n(i)}=\eta W_{t}
$$

where $\eta$ is a positive constant, and $\eta \leq 1$.

${ }^{1}$ KHOA website: http://www.khoa.go.kr

${ }^{2}$ NOAA website: http://tidesandcurrents.noaa.gov
Assuming the maximum speed and the angular range of the operation, the linear and angular motion can be achieved independently. The linear and angular control laws are formulated as:

$$
\begin{gathered}
u_{t o l}=\left\{\begin{array}{l}
k_{u} F_{s y n}, u_{t o l} \leq u_{\max } \\
u_{\max }, u_{t o l}>u_{\max }
\end{array}\right. \\
r=\left\{\begin{array}{c}
k_{r}\left(\theta_{F_{s y n}}-\psi\right), r \leq r_{\max }, \\
r_{\max }, r>r_{\max },
\end{array}\right.
\end{gathered}
$$

where $k_{u}, k_{r}$ are the gain coefficients, $u_{\max }, r_{\max }$ are the maximum values of the resultant velocity and angular velocity, and $\theta_{F_{s y n}}$ is the angle between the direction of the synthetic force and the USV heading.

Fig. 4 depicts the synthetic force function $\left(F_{s y n}\right)$ with different force weights $\varepsilon_{1}$ and $\varepsilon_{2}$, which affects the USF path distance and energy consumption for local path planning. For convenience, we set up an obstacle-free simulation environment, where the color varying from blue to red represents different potential values from low to high as shown in the bar chart. For the simulation parameters, refer to Section 4.

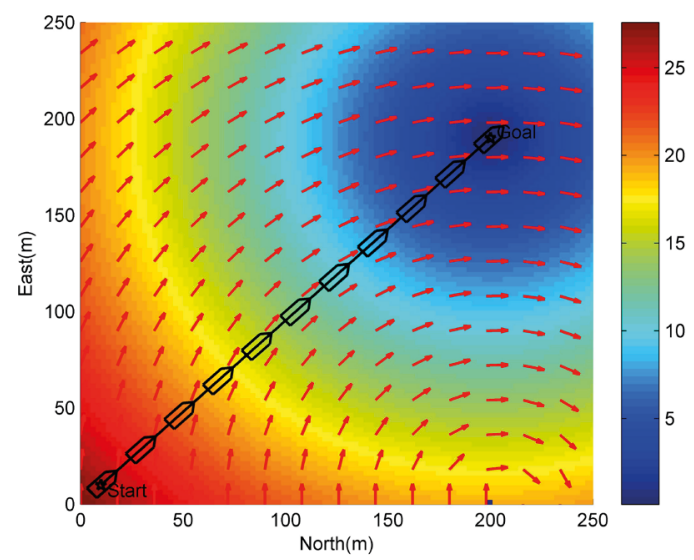

(a) $\varepsilon_{1}=0, \varepsilon_{2}=1$

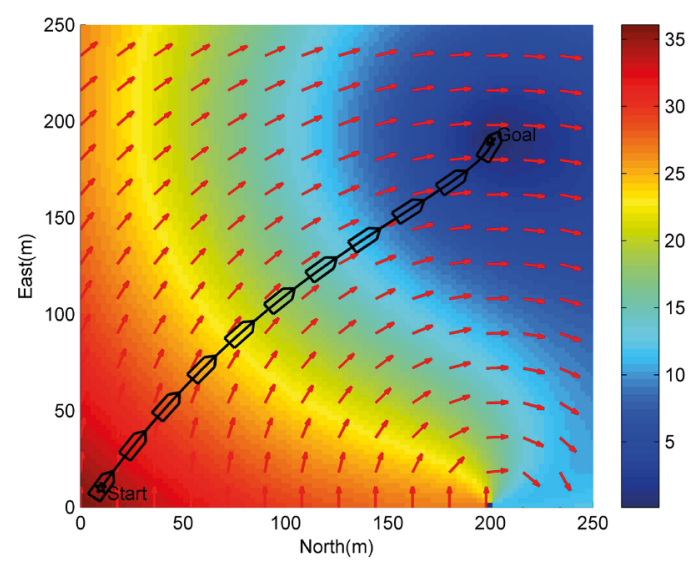

(b) $\varepsilon_{1}=0.004, \varepsilon_{2}=1$ 


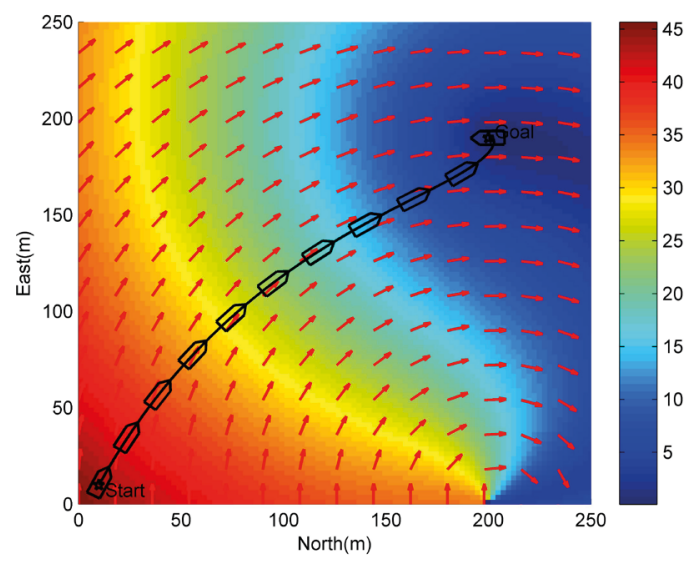

(c) $\varepsilon_{1}=0.008, \varepsilon_{2}=1$

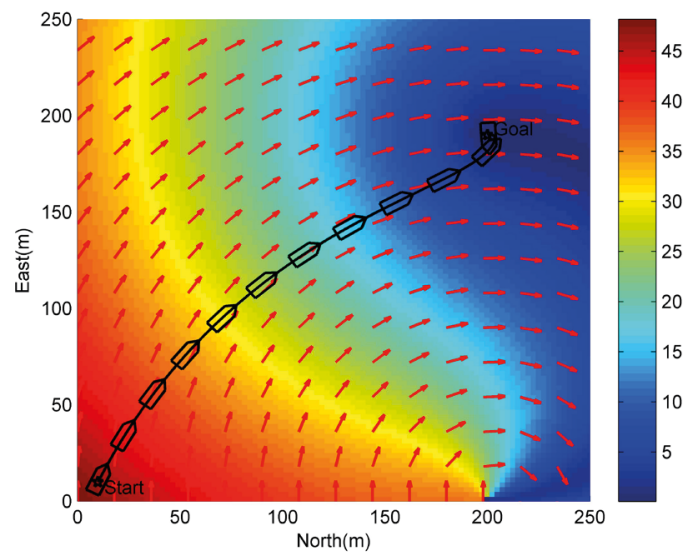

(d) $\varepsilon_{1}=0.009, \varepsilon_{2}=1$

Fig. 4. Simulations with different force weights

Tab.1. Energy consumption and travel distance for different force weights

\begin{tabular}{|c|c|c|c|}
\hline$\varepsilon_{1}, \varepsilon_{2}$ & $\begin{array}{c}\text { Energy } \\
\text { consumption } \\
\boldsymbol{W}_{\text {syn }}(\mathrm{J})\end{array}$ & $\begin{array}{c}\text { Actual } \\
\text { Energy } \\
\text { consumption } \\
\boldsymbol{W}_{\boldsymbol{t}}(\mathrm{J})\end{array}$ & $\begin{array}{c}\text { Travel } \\
\text { Distance (m) }\end{array}$ \\
\hline$\varepsilon_{1}=0, \varepsilon_{2}=1$ & 4685469 & 5512316 & 262 \\
\hline$\varepsilon_{1}=0.004, \varepsilon_{2}=1$ & 4568981 & 5375272 & 265 \\
\hline$\varepsilon_{1}=0.008, \varepsilon_{2}=1$ & 4435467 & 5218196 & 272 \\
\hline$\varepsilon_{1}=0.009, \varepsilon_{2}=1$ & 4538579 & 5339504 & 274 \\
\hline
\end{tabular}

When the marine environment is neglected, the shortest distance corresponds to the minimum energy consumption paths, otherwise the shortest distance and the minimum energy consumption paths can be different. From the simulation results shown in Fig. 4 and the data analysis of Tab.1, the influence of different force weighs on the travel distance and the energy consumption of the planned path can be obtained. When neglecting the wind and surface currents, i.e. $\varepsilon_{1}=0, \varepsilon_{2}=1$, the planned path is the shortest and the energy consumption reaches minimum. On the other hand, when considering the wind and the surface currents, the planned path has the minimum energy consumption for $\varepsilon_{1}=0.008, \varepsilon_{2}=1$, but the travel distance is not the shortest for these values. In order to achieve the minimum energy consumption, force weighting is used. The relationship between the actual energy consumption $W_{t}$ and the energy consumption $W_{\text {syn }}$ is $\eta=0.85$.

\section{SIMULATIONS}

The proposed multi-layered potential field method for local path planning has been tested and verified in different marine environments subject to wind and surface currents. For clarity, the simulation results are compared with the situation which neglects the wind and currents. Performance evaluations of the proposed algorithm and the planned USV path are provided in Figs. 5-7 and Tab.2 In the simulation environment, the dimension of the simulation area is $250 \mathrm{~m} \times 250 \mathrm{~m}$, while the initial point and the target point of the USV voyage are $(10 m, 10 m),(200 m, 190 m)$, respectively. The concentric circle is a dynamic obstacle, and the outer circle is its influence range which is 3 times the length of the dynamic obstacle. Besides, the initial point and the target point of USV voyage are marked as black stars, while the instantaneous USV positions on the planned path are drawn as black solid line markers. The vector arrows represent the size and direction of the resultant forces of the wind and surface currents. The resultant direction of the wind and surface currents is gradually shifted eastward. The multilayered potential field generated in the simulations is shown using the color which varies from blue to red to represent different potential values from low to high as shown in the bar chart. It can also be observed that the target point has the lowest potential field. Before starting the simulations, the following assumptions have been made:

1) The kinetic equation is not considered, and the USV only considers kinematic equations.

2) The water in the environment is assumed to have a constant density, $\rho=$ const.

3) The wind and surface currents have the same direction, and the initial direction of the wind $\varphi_{w o}$ is south.

The simulation parameters are defined as follows. $g=9.8 \mathrm{~m} / \mathrm{s}, \quad \theta= \pm 120^{\circ}, \rho=1025 \mathrm{~kg} / \mathrm{m}^{3}, \rho_{a}=1.29 \mathrm{~kg} / \mathrm{m}^{3}$, $V_{w}=10 \mathrm{~m} / \mathrm{s}, \varphi_{w 0}=90^{\circ}, \alpha=800, \beta=9.6, \lambda_{2}=40, \lambda_{3}=35$, $\lambda_{4}=2, d_{0}=30 \mathrm{~m}, u_{\max }=10 \mathrm{~m} / \mathrm{s}, k_{u}=0.0018, r_{\text {max }}=0.4 \mathrm{rad} / \mathrm{s}$ , $k_{r}=0.0053$.

It should be noted that these parameters can be adapted to different environmental situations.

Remark 1: For interpretation of the references to colors in all figure legends, the reader is referred to the web version of this article.

Tab. 2. Energy consumption and distance in different dynamic environments

\begin{tabular}{|c|c|c|}
\hline$\varepsilon_{\mathbf{1}}=\mathbf{0 . 0 0 8}, \varepsilon_{\mathbf{2}}=\mathbf{1}$ & Energy consumption $\boldsymbol{W}_{\text {syn }}(\mathrm{J})$ & Travel Distance $(\mathrm{m})$ \\
\hline Fig. 5 (a) & 4851863 & 275 \\
\hline Fig. 5 (b) & 4698202 & 273 \\
\hline Fig. 5 (c) & 4869640 & 275 \\
\hline
\end{tabular}




\begin{tabular}{|c|c|c|}
\hline$\varepsilon_{\mathbf{1}} \mathbf{=} \mathbf{0 . 0 0 8}, \varepsilon_{\mathbf{2}}=\mathbf{1}$ & Energy consumption $\boldsymbol{W}_{\text {syn }}(\mathrm{J})$ & Travel Distance (m) \\
\hline Fig. 5 (d) & 4774198 & 278 \\
\hline Fig. 6 (a) & 4867783 & 275 \\
\hline Fig. 6 (b) & 4716429 & 274 \\
\hline Fig. 6 (c) & 4747234 & 272 \\
\hline Fig. 6 (d) & 4591421 & 265 \\
\hline Fig. 7 (a) & 4845201 & 274 \\
\hline Fig. 7 (b) & 4751472 & 276 \\
\hline Fig. 7 (c) & 5145850 & 271 \\
\hline Fig. 7 (d) & 4727282 & 293 \\
\hline
\end{tabular}

To validate the performance of the proposed algorithm, simulation studies were performed in different dynamic environments, including overtaking and crossing situations. The multi-layered potential field method was then run in the same dynamic obstacle environments using the same simulation parameters, and start and target points of the mission. The only difference in the comparison simulations was whether the simulation took into account, or neglected,

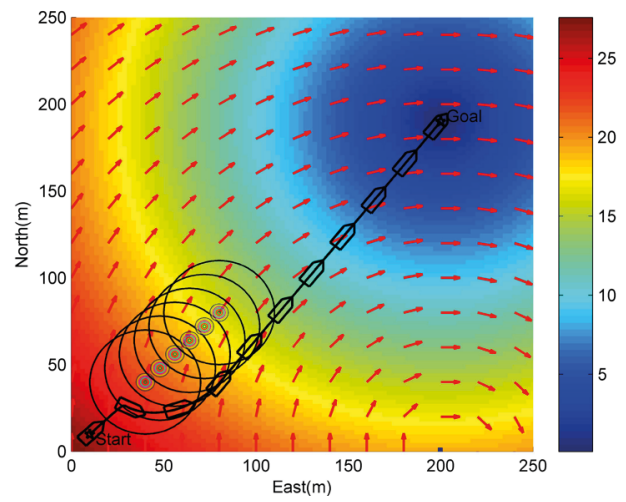

a)

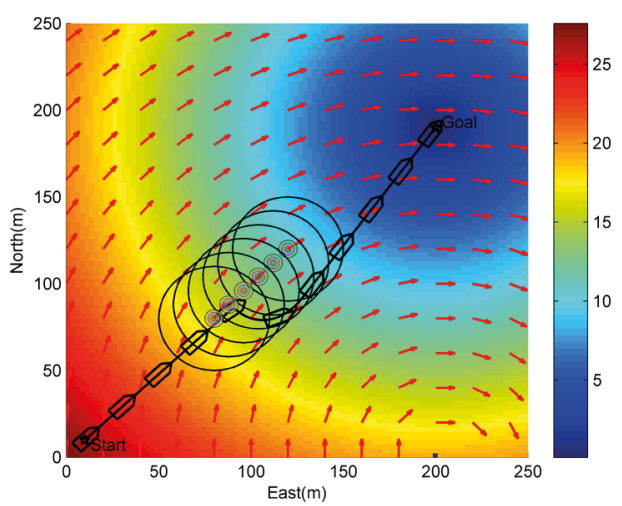

c) the influence of wind and surface currents. The simulation results are shown in Figs. 5-7, which include a single dynamic obstacle and multiple dynamic obstacles in overtaking and crossing situations. The simulations of single dynamic obstacle overtaking and crossing situations are shown in Figs. 5-6, in which the effect of wind and surface currents is considered in Figs. (b) and (d), and neglected in Figs. (a) and (c)). Multiple dynamic obstacle simulation results are shown in Fig. 7, where the wind and surface currents are considered in (b) and (d). To further compare different simulation results, quantitative evaluations of the planned paths are given in Tab. 2. These data indicate that local path planning making use of the multi-layered potential field method can save on the energy cost while keeping the distance cost as low as possible. It can be seen that the energy consumption along the planned path under the environmental interference is relatively small, which proves the effectiveness of the proposed algorithm. Meanwhile, the above planned paths are able to successfully avoid dynamic obstacles.

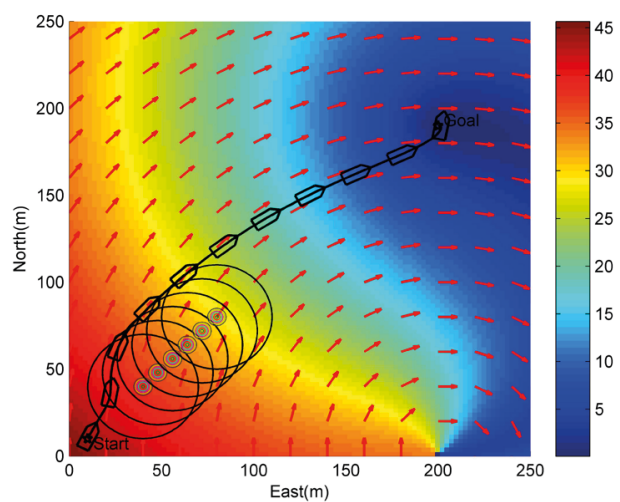

b)

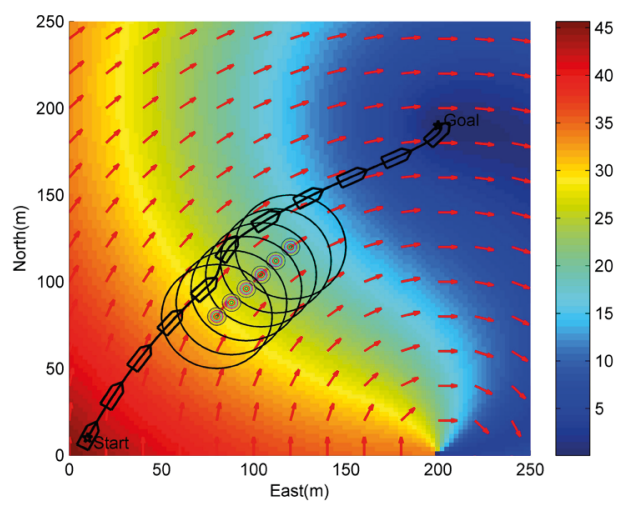

d)

Fig. 5. Simulation results using multi-layered potential field method in a single dynamic obstacle-overtaking situation, while considering the effect of wind and surface currents - cases (b) and (d), and neglecting it - cases (a) and (c). 


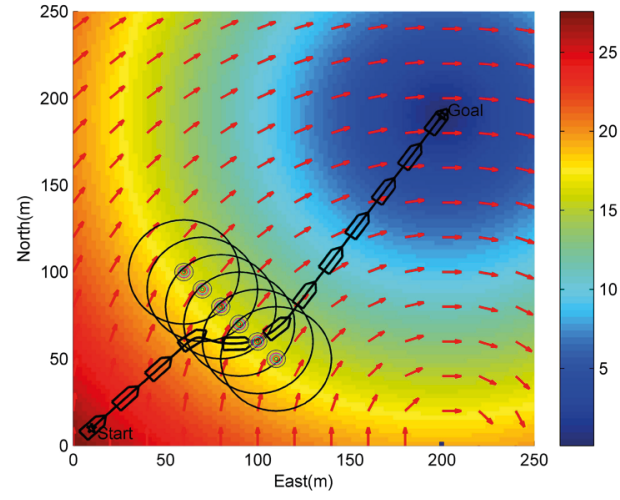

a)

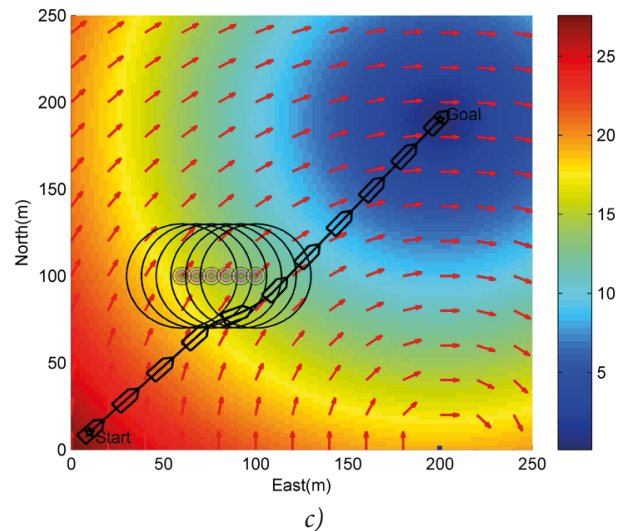

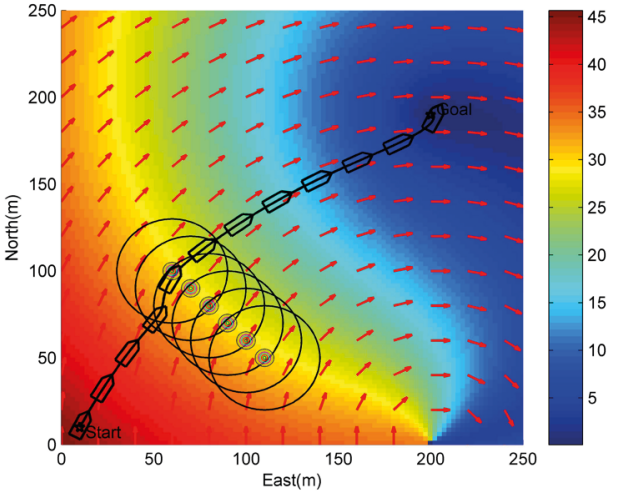

b)

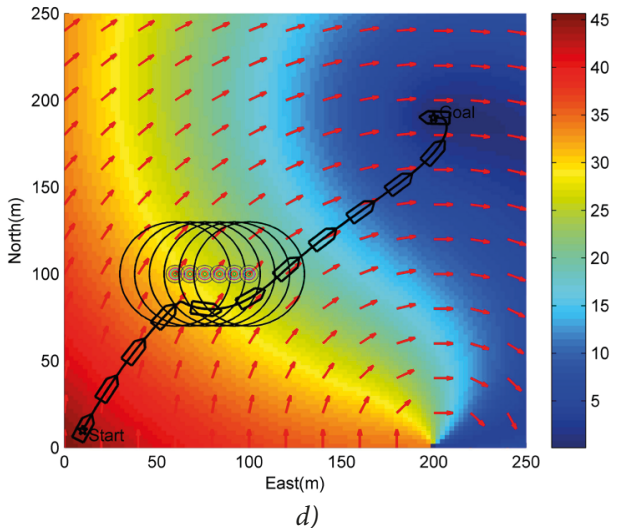

Fig. 6. Simulation results using multi-layered potential field method in a single dynamic obstacle-crossing situation, while considering the effect of wind and surface currents - cases $(b)$ and $(d)$, and neglecting it - cases (a) and (c).

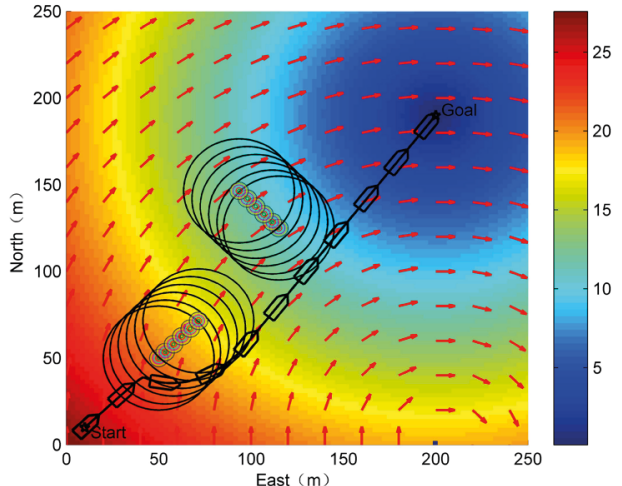

a)

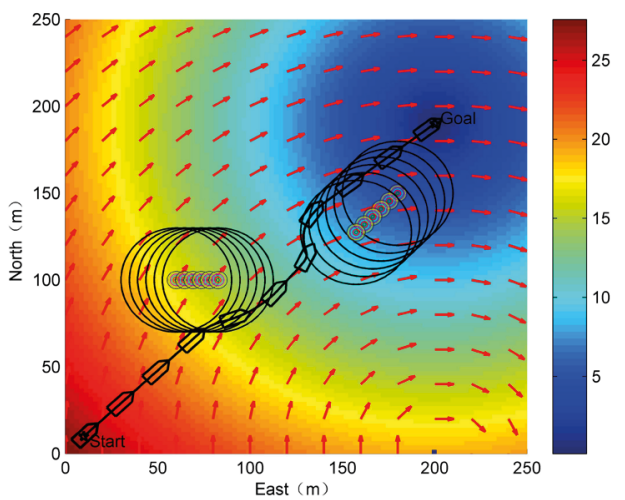

c)

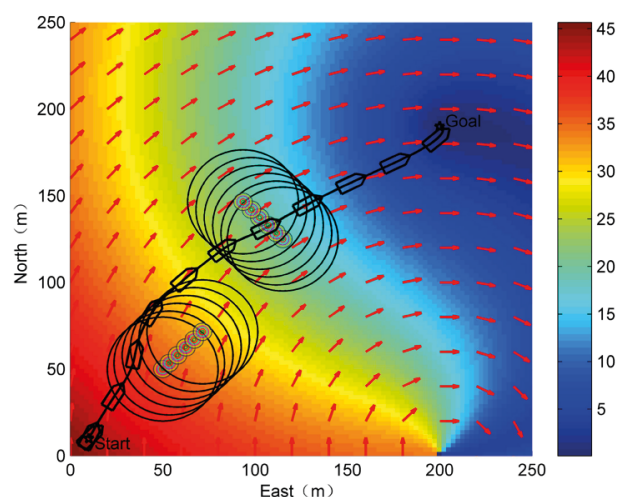

b)

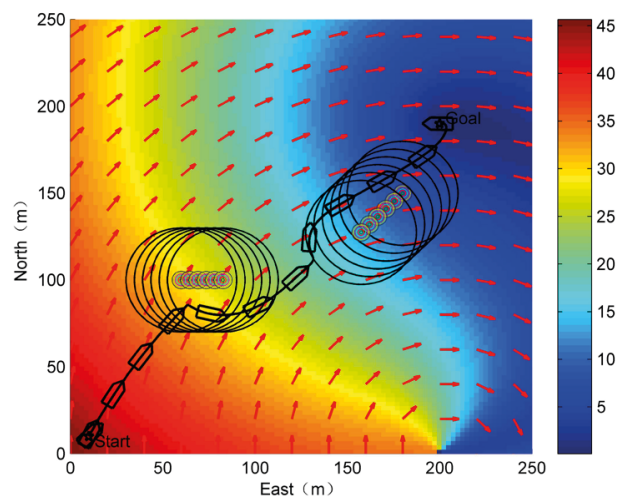

d)

Fig. 7. Simulation results using multi-layered potential field method for multiple dynamic obstacles- overtaking and crossing situation, while considering the effect of wind and surface currents - cases $(b)$ and $(d)$, and neglecting it - cases $(a)$ and $(c)$. 


\section{CONCLUSIONS AND FUTURE PLANS}

The multi-layered potential field method has been used for local path planning of a water-jet propelled USV in the presence of wind and surface currents, in order to achieve minimum energy consumption. Comparing to the previous researches, the proposed method constructs a synthetic environment framework, which incorporates the information of the base potential field layer and the environment layer. In addition, the traditional potential field method integrates kinematic control and is extended by including the velocity information of the USV and the obstacles to avoid the collision with dynamic obstacles. The performance of the proposed algorithm has been positively verified through comparison of simulation results, thus confirming the reliability and efficiency of the algorithm in avoiding dynamic obstacles and minimizing energy consumption.

For future work, the proposed algorithm will be improved in several ways to increase further the practicability of the planned paths. Firstly, it will undergo verifications in reallife experiments with physical USV models and, after that, in a real environment on board a USV to demonstrate its feasibility. Secondly, the hydrodynamic forces will be combined with the kinetic equation to consider their influence on USV performance. Thirdly, the developed method can be further extended to handle more complex cases, such as to track the moving target. From a general point of view, these future works will contribute to the development of autonomy of USV operation, which is also the ultimate goal of this research work.

\section{APPENDIX A}

Under three degrees of freedom, the force of wind and surface currents is represented by [26]:

$$
\begin{gathered}
\tau_{\text {wind }}=\left[\begin{array}{lll}
X_{\text {wind }}, & Y_{\text {wind }}, & N_{\text {wind }}
\end{array}\right]^{T} \\
\tau_{\text {current }}=\left[\begin{array}{lll}
X_{\text {current }}, & Y_{\text {current }}, & N_{\text {current }}
\end{array}\right]^{T}
\end{gathered}
$$

and,

$$
\begin{aligned}
& \left\{\begin{array}{l}
X_{\text {wind }}=\frac{1}{2} \rho_{a} V_{w}^{2} C_{X w}\left(\gamma_{w}\right) A_{f} \\
Y_{\text {wind }}=\frac{1}{2} \rho_{a} V_{w}^{2} C_{Y w}\left(\gamma_{w}\right) A_{s} \\
N_{\text {wind }}=\frac{1}{2} \rho_{a} V_{w}^{2} C_{N w}\left(\gamma_{w}\right) A_{s} L_{o a}
\end{array}\right. \\
& \left\{\begin{array}{l}
X_{\text {current }}=\frac{1}{2} \rho V_{c} C_{X c}\left(\mu_{c r}\right) A_{T C} \\
Y_{\text {current }}=\frac{1}{2} \rho V_{c} C_{Y c}\left(\mu_{c r}\right) A_{L S} \\
N_{\text {current }}=\frac{1}{2} \rho V_{c} C_{N c}\left(\mu_{c r}\right) A_{L S} \cdot L_{o a}
\end{array}\right.
\end{aligned}
$$

where $\rho_{a}$ is the air density; $C_{X_{w}}\left(\gamma_{w}\right), C_{Y_{w}}\left(\gamma_{w}\right), C_{N_{w}}\left(\gamma_{w}\right)$ and

\begin{tabular}{|c|c|c|}
\hline Property (Unit) & Symbol & Value \\
\hline Quality (Kg) & $m$ & 5540 \\
\hline Length (m) & $L_{o a}$ & 9.5 \\
\hline Width (m) & $B$ & 2.8 \\
\hline Depth (m) & $D$ & 1.43 \\
\hline Tilt angle ( $\bigcirc$ ) & $\beta$ & 25.6 \\
\hline Cross sectional diameter of inlet water $(\mathrm{m})$ & $D_{i n}$ & 0.223 \\
\hline Cross sectional diameter of nozzle $(\mathrm{m})$ & $D_{j}$ & 0.182 \\
\hline Cross sectional diameter of bend $(\mathrm{m})$ & $D_{w}$ & 0.23 \\
\hline Distance between center of gravity and stern $(\mathrm{m})$ & $X_{C G}$ & 3.26 \\
\hline Forward projection area above waterline $\left(\mathrm{m}^{2}\right)$ & $A_{f}$ & 3.76 \\
\hline Lateral projection area above waterline $\left(\mathrm{m}^{2}\right)$ & $A_{s}$ & 13.41 \\
\hline Forward projection area below waterline $\left(\mathrm{m}^{2}\right)$ & $A_{T C}$ & 1.32 \\
\hline Lateral projection area below waterline $\left(\mathrm{m}^{2}\right)$ & $A_{L S}$ & 8.97 \\
\hline Energy loss coefficient of inlet (NA) & $e_{i n}$ & 0.03 \\
\hline Energy loss coefficient of bend (NA) & $e_{w}$ & 0.06 \\
\hline Energy loss coefficient of nozzle (NA) & & 0.03 \\
\hline Transmission efficiency of drive shaft (NA) & $\eta_{s}$ & 0.95 \\
\hline The efficiency of the propeller (NA) & $\eta_{p}$ & 0.90 \\
\hline Propeller absorption Power (1000r/min) ( NA) & $C$ & 27.13 \\
\hline
\end{tabular}
$C_{X c}\left(\mu_{c r}\right), C_{Y c}\left(\mu_{c r}\right), C_{N c}\left(\mu_{c r}\right)$ are the wind force coefficients and the surface current force coefficients, respectively.

Tab. 3. Parameters of water-jet propelled unmanned surface vehicle

\section{REFERENCES}

1. Liu Z, Zhang Y, Yu X, et al.: Unmanned surface vehicles: An overview of developments and challenges. Annual Reviews in Control, vol.41, pp:71-93, 2016.

2. Lazarowska, Agnieszka.: A new deterministic approach in a decision support system for ship's trajectory planning. Expert Systems With Applications, vol.71, pp:469-478, 2017.

3. Murphy R, Steimle E, Griffin C, et al. Cooperative use of unmanned sea surface and micro aerial vehicles at Hurricane Wilma. Journal of Field Robotics, 25(3):164-180, 2008. 
4. Lazarowska A.: Swarm Intelligence Approach to Safe Ship Control. Polish Maritime Research, 22(4):34-40, 2015.

5. Li W, Ma W.: Simulation on Vessel Intelligent Collision Avoidance Based on Artificial Fish Swarm Algorithm. Polish Maritime Research, 23, 2016.

6. Shen Y, Zhao N, Xia M, et al.: A Deep Q-Learning Network for Ship Stowage Planning Problem. Polish Maritime Research, 24, 2017.

7. Chen D Z, Szczerba R J, Uhran J.: Planning conditional shortest paths through an unknown environment: a framed-quadtree approach. 1995.

8. Kamon I, Rivlin E.: Sensory based motion planning with global proofs. International Conference on Intelligent Robots and Systems. IEEE Computer Society, 1995.

9. Svec P, Gupta S K.: Automated synthesis of action selection policies for unmanned vehicles operating in adverse environments. Autonomous Robots 149-164, 2012.

10. Goldberg D E.: Genetic Algorithms in Search, Optimization and Machine Learning. xiii(7): 2104-2116,1989.

11. Petres C, Yan P, Patron P, et al.: Path Planning for Autonomous Underwater Vehicles. IEEE Transactions on Robotics, 23(2):331-341, 2007.

12. Lavalle S M.: Rapidly-Exploring Random Trees: A New Tool for Path Planning. Algorithmic \& Computational Robotics New Directions, 293-308, 1998.

13. Ge S, Cui Y J.: Dynamic Motion Planning for Mobile Robots Using Potential Field Method. Autonomous Robots, 13(3):207-222, 2002.

14. Andrews J R, Hogan N.: Impedance Control as a Framework for Implementing Obstacle Avoidance in a Manipulator. Control of Manufacturing Processes and Robotic Systems. 243-251,1983.

15. Zhu Y, Zhang T, Song J.: Study on the Local Minima Problem of Path Planning Using Potential Field Method in Unknown Environments. Acta Automatica Sinica, vol.36(8), pp:1122-1130, 2010.

16. Liu Y, Song R, Bucknall R. A practical path planning and navigation algorithm for an unmanned surface vehicle using the fast marching algorithm. Oceans. IEEE, 2015.

17. Zhu Y, Zhang T, Song J.: Path planning for nonholonomic mobile robots using artificial potential field method. Control Theory \& Applications, 27(2):152-158, 2010.
18. Kovács B, Szayer G, Tajti F, et al.: A novel potential field method for path planning of mobile robots by adapting animal motion attributes. Robotics \& Autonomous Systems, 82(C):24-34, 2016.

19. Lee T H, Chung H, Myung H.: Multi-resolution path planning for marine surface vehicle considering environmental effects. Oceans. IEEE, 2011.

20. Garau B, Alvarez A, Oliver G.: Path Planning of Autonomous Underwater Vehicles in Current Fields with Complex Spatial Variability: an $A^{\star}$ Approach, 2005.

21. Isern-González J, Hernández-Sosa D, Fernández-Perdomo E, et al.: Path planning for underwater gliders using iterative optimization. IEEE International Conference on Robotics and Automation, 1538-1543, 2011.

22. Soulignac M.: Feasible and Optimal Path Planning in Strong Current Fields. IEEE Transactions on Robotics, 27(1):89-98, 2011.

23. Lee T, Kim H, Chung H, et al.: Energy efficient path planning for a marine surface vehicle considering heading angle. Ocean Engineering, 107:118-131, 2015.

24. Song R, Liu Y, Bucknall R.: A multi-layered fast marching method for unmanned surface vehicle path planning in a time-variant maritime environment. Ocean Engineering, 129:301-317, 2017.

25. Gong-Xing W U, Jin Z, Lei W, et al.: Design of the basic motion control system for water-jet-propelled unmanned surface vehicle. Control Theory \& Applications, 27, 2010.

26. Yue J.: Study on Modeling and Simulation of 6-DOF Motion of Water-jet Propelled Unmanned Surface Vehicle. Dalian Maritime University, 17-60, 2016.

\section{CONTACT WITH THE AUTHORS}

\author{
Shasha Wang \\ e-mail:wangshasha@hrbeu.edu.cn
}

Harbin Engineering University No.145 Nantong Street, Nangang District 15001 Harbin

China 